\title{
Review of individual placement and support (IPS) studies and results on health status of people with long-term mental disorder and competitive employment
}

\author{
Francisco Rodríguez Pulido ${ }^{1 *}$, Nayra Caballero Estebaranz ${ }^{2}$, Dácil Oramas Pérez ${ }^{3}$ and Celia León Palacín ${ }^{4}$ \\ ${ }^{1}$ Psychiatrist, Department of Psychiatry, University of La Laguna. Insular Plan of Psychosocial Rehabilitation, Spain \\ ${ }^{2}$ Individual Placement and Support (IPS) Team for people with severe mental illness, SINPROMI, Spain \\ ${ }^{3}$ Department of Family and Community Medicine, Canary Health Service, Spain \\ ${ }^{4}$ Psychologist, Canarian Creative Therapies Association, Spain
}

\begin{abstract}
Ordinary employment, from the vulnerability-stress model, is believed to trigger relapses in people with long-term mental disorders when subjected to demanding work environments. To test the accuracy of this hypothesis, different databases between 1998 and 2019 (May) were consulted, using various key words. Randomized Clinical Trials (RCTs) that analyzed non-vocational outcomes related to symptomatology and hospitalizations in the Individual Placement and Support (IPS) strategy with severe mental disorders were specifically reviewed. A total of 383 references were reviewed, 26 were selected and 18 were included. Of the selected studies the follow-up period is between 12 months and 24 months for the most part. Samples usually range from $100-200$ participants but there are studies with larger samples, one study with over 2059 participants. The most commonly used outcome is admissions and relapses, the most commonly used being days of hospitalization. The most widely used scales were on overall functioning, GAF and to measure relapse, PANNS and BPRS. Competitive employment was found not to cause relapses or hospitalisations, and long-term employment seemed to contribute to a favourable clinical evolution, although the degree of impact on the health status has yet to be proven.
\end{abstract}

\section{Introduction}

As adults, people must make their own decisions, take responsibility and assume the consequences of these decisions on an equal footing with other citizens. This makes employment one of the main routes to social integration for recovering people with long-term mental illness. These processes facilitate the significance of their role as citizens and recover their meaning in clinical and personal recovery. In the United States, Europe and Asia, new initiatives have been developed such as the Individual Placement and Support (IPS), which is based primarily on the placement of people with long-term mental illness into competitive jobs with ongoing support follow-up, in contrast to traditional vocational services that use "train and then place": assessments, training skills, counseling, sheltered work experiences and job adjustments. IPS studies over the past 20 years have primarily focused on vocational outcomes [1-4]. In this line, in Tenerife, in a context of high unemployment, we have developed the IPS strategy [5-7].

On the other hand, there is literature on ordinary employment for people with long-term mental disorders based on the belief that ordinary employment, from the vulnerability-stress model, can trigger relapses of their illness when they are subjected to an environment that generates stressful situations, both because of the demands of their work and because of their relationships with their colleagues and bosses [8]. Not all studies using the IPS strategy publish data on their non-vocational outcomes [9-11], but when they do, relapses (severity of symptoms and/or hospitalization during employment) [12], self-esteem and quality of life have been reported $[13,14]$. An increasing number of studies, apart from the vocational impact, analyze how employment influences a broader part of people's lives that includes aspects of their health.

Many people with long-term mental illness emphasize the key role of work in their recovery processes [13,15-17]. Employment has multiple economic, social and psychological benefits. Generating one's own income gives individuals a sense of stability and direction in life, a sense of belonging and personal identity, generating and enhancing interpersonal relationships and communications, and helping to structure time [18] as well as enhance autonomy. This autonomy implies managing one's own affairs without supervision or control by others; it implies freedom and the ability to choose, being intimately associated with integration into the community [19], because most users, if given the opportunity, prefer to have the freedom to live, work and relate to a variety of people in the community.

The aim of this review is to analyse randomised clinical trials (RCTs) with the IPS strategy in relation to their non-vocational outcomes (symptoms and hospitalisations), which have been published in the scientific literature during the 1998-2019 period.

*Correspondence to: Francisco Rodríguez Pulido, MD Ph.D. Psychiatrist, Department of Psychiatry, University of La Laguna. Insular Plan of Psychosocial Rehabilitation, Spain, E-mail: fpulido15@yahoo.es

Key words: clinical functioning, employment, evaluation, non-vocational outcomes, psychiatric hospitalizations, psychological symptoms, rehabilitation, schizophrenia

Received: May 21, 2020; Accepted: June 12, 2020; Published: June 16, 2020 
Pulido FR (2020) Review of individual placement and support (IPS) studies and results on health status of people with long-term mental disorder and competitive employment

\section{Materials and methods}

An internet search of the following databases was conducted: MEDLINE, EMBASE, SCI, PreMedline, CRD, Cochrane Library, CINAHL, PsyclNFO from 1998 to May 2019 containing the following keywords: supported employment, clinical functioning, individual placement and support, psychological symptoms, psychiatric hospitalizations and schizophrenia. RCTs published in English during the selected period were chosen. To meet the stated objectives, a detailed protocol was developed, which describes the following stages of the systematic search process: 1) definition of the selection criteria (inclusion and exclusion criteria), 2) search for relevant published RCT articles, 3 ) selection of titles and abstracts that meet the selection criteria, 4) review of full articles representing the potentially selected studies, 5) critical appraisal of the quality of the selected studies and the extraction of data of interest, and 6) data analysis and synthesis.

Three reviewers conducted the entire study selection process. They analysed the studies separately and then shared their results. If there was any doubt and/or disagreement between them, a fourth reviewer would check the protocol criteria and try to reach a consensus with the other three reviewers to see if the study selection was appropriate.

\section{Inclusion and exclusion criteria}

The included studies were RCTs. Cohort studies, systemic reviews, comparative, observational, economic evaluation, qualitative, historical reviews, case studies or expert consensus studies were excluded.

\section{Participants}

Studies including participants with the following criteria were included:

a) Studies that followed the intervention according to the IPS model and met its principles

b) Persons with severe and persistent mental disorder (SMD) (National Institute of Mental Health [NIMH]).

c) Ages between 18 and 65 years.

d) Studies whose participants were actively dependent on substances as long as they had SMD as their primary diagnosis.

(e) Studies measuring the symptomatology variable or hospitalizations with the IPS strategy.

\section{Measuring instrument}

Different scales were used to measure the symptomatology variables (Tables 1-3). They normally provide an overall score and their cut-off points serve to identify the severity of the disorder. Each scale has its cut-off points and above these, it is usually used as a criterion for relapse, compared with the baseline.

To measure overall functioning the most commonly used scale is the Global Assessment of Functioning (GAF) or Global Assessment Scale (GAS). This variable was measured in eight studies in this review [10,20-26]. The World Health Organization Disability Assessment Schedule (WHODAS) was also used by one study [27]. The remaining studies did not report on this variable.

One of the outcomes on relapse used was days of hospitalization (Table 4). This data is reflected in 14 of the RCT studies analysed [2024,28-36].
Table 1. Study groups and sample size (ACT: Assertive Community Treatment. CVR Conventional Vocational Rehabilitation. DPA: Diversified Placement Approach. EVR Enhanced Vocational Rehabilitation. IPS: Individual Placement Support. ISE: Integrated Supported Employment. PSR: PsychoSocial Rehabilitation. SAU: Services As Usual SE: Supported Employment. SEP: Supported Employment Program. TVR: Traditional Vocational Rehabilitation. US: Usual Services. VR: Vocational Rehabilitation. VS: Vocational Service)

\begin{tabular}{|c|c|}
\hline Study & Sample size \\
\hline Drake, 1999 & $\begin{array}{c}\operatorname{IPS}(n=76) \\
\operatorname{EVR}(n=76) \\
\quad N=152\end{array}$ \\
\hline Bond, 2001 & $\begin{array}{l}\text { Competitive work } \\
\text { Sheltered work } \\
\text { Minimal work } \\
\text { No work } \\
\mathrm{N}=149\end{array}$ \\
\hline Mueser, 2004 & $\begin{array}{c}\text { IPS }(\mathrm{n}=68) \\
\text { PSR }(\mathrm{n}=67) \\
\text { Standard service }(\mathrm{n}=69) \\
\mathrm{N}=204\end{array}$ \\
\hline Gold, 2006 & $\begin{array}{c}\mathrm{SE}(\mathrm{n}=77) \\
\mathrm{ACT}+\mathrm{IPS}(\mathrm{n}=66) \\
\mathrm{N}=143\end{array}$ \\
\hline Latimer, 2006 & $\begin{array}{c}\text { SE }(n=75) \\
\text { US }(n=74) \\
N=149\end{array}$ \\
\hline Bond, 2007 & $\begin{array}{c}\text { IPS }(\mathrm{n}=92) \\
\text { DPA }(\mathrm{n}=95) \\
\mathrm{N}=187\end{array}$ \\
\hline Burns, 2007 & $\begin{array}{l}\text { IPS }(n=148) \\
\text { VS }(n=141) \\
N=289\end{array}$ \\
\hline Burns, 2008 & $\begin{array}{l}\text { IPS }(n=148) \\
\text { VS }(n=141) \\
N=289\end{array}$ \\
\hline Wong, 2008 & $\begin{array}{c}\mathrm{SE}(\mathrm{n}=46) \\
\operatorname{CVR}(\mathrm{n}=46) \\
\mathrm{N}=92\end{array}$ \\
\hline Burns, 2009 & $\begin{array}{l}\text { IPS }(n=156) \\
\text { VS }(n=156) \\
N=312\end{array}$ \\
\hline Nygren, 2011 & $\begin{array}{c}\text { IPS }(\mathrm{n}=65) \\
\mathrm{N}=65\end{array}$ \\
\hline Kilian, 2012 & $\begin{array}{c}\text { IPS }(n=156) \\
\text { VS }(n=156) \\
N=312\end{array}$ \\
\hline Drake, 2013 & $\begin{array}{c}\text { IPS }(n=892) \\
\text { SAU }(n=973) \\
N=1.865\end{array}$ \\
\hline Kukla, 2013 & $\begin{array}{c}\text { IPS }(n=92) \\
\text { DPA }(n=95) \\
N=187\end{array}$ \\
\hline Bejerlhom, 2014 & $\begin{array}{c}\text { IPS }(n=60) \\
\operatorname{TVR}(n=60) \\
N=120\end{array}$ \\
\hline Hoffman, 2014 & $\begin{array}{c}\operatorname{SEP}(n=46) \\
\operatorname{TVR}(n=54) \\
N=100\end{array}$ \\
\hline Zhang, 2017 & $\begin{array}{c}\text { ISE }(\mathrm{n}=54) \\
\operatorname{IPS}(\mathrm{n}=54) \\
\text { TVR }(\mathrm{n}=54) \\
\mathrm{N}=162\end{array}$ \\
\hline Reme, 2019 & $\begin{array}{c}\text { IPS }(\mathrm{n}=227) \\
\operatorname{VR}(\mathrm{n}=181) \\
\mathrm{N}=408\end{array}$ \\
\hline
\end{tabular}


Pulido FR (2020) Review of individual placement and support (IPS) studies and results on health status of people with long-term mental disorder and competitive employment

Table 2. Follow-up period

\begin{tabular}{|c|c|}
\hline Study & Follow-up \\
\hline Drake, 1999 & $\begin{array}{l}\text { Baseline } \\
6 \text { months } \\
12 \text { months } \\
18 \text { months }\end{array}$ \\
\hline Bond, 2001 & 18 months \\
\hline Mueser, 2004 & $\begin{array}{l}\text { Baseline } \\
6 \text { months } \\
12 \text { months } \\
18 \text { months } \\
24 \text { months }\end{array}$ \\
\hline Gold, 2006 & $\begin{array}{l}\text { Baseline } \\
6 \text { months } \\
12 \text { months } \\
18 \text { months } \\
24 \text { months }\end{array}$ \\
\hline Latimer, 2006 & $\begin{array}{l}\text { Baseline } \\
6 \text { months } \\
12 \text { months }\end{array}$ \\
\hline Bond, 2007 & $\begin{array}{l}\text { Baseline } \\
6 \text { months } \\
12 \text { months } \\
18 \text { months } \\
24 \text { months }\end{array}$ \\
\hline Burns, 2007 & $\begin{array}{l}\text { Baseline } \\
6 \text { months } \\
12 \text { months } \\
18 \text { months }\end{array}$ \\
\hline Burns, 2008 & 18 months \\
\hline Wong, 2008 & $\begin{array}{l}\text { Baseline } \\
6 \text { months } \\
12 \text { months } \\
18 \text { months }\end{array}$ \\
\hline Burns, 2009 & $\begin{array}{l}\text { Baseline } \\
6 \text { months } \\
12 \text { months } \\
18 \text { months }\end{array}$ \\
\hline Nygren, 2011 & $\begin{array}{l}\text { Baseline } \\
12 \text { months } \\
24 \text { months }\end{array}$ \\
\hline Kilian, 2012 & $\begin{array}{c}\text { Baseline } \\
6 \text { months } \\
12 \text { months } \\
18 \text { months }\end{array}$ \\
\hline Drake, 2013 & $\begin{array}{c}\text { Baseline } \\
\text { Every month up to } 24 \text { months }\end{array}$ \\
\hline Kukla, 2013 & $\begin{array}{l}\text { Baseline } \\
6 \text { months } \\
12 \text { months } \\
18 \text { months } \\
24 \text { months }\end{array}$ \\
\hline Bejerlhom, 2014 & $\begin{array}{l}\text { Baseline } \\
6 \text { months } \\
18 \text { months }\end{array}$ \\
\hline Hoffman, 2014 & $\begin{array}{c}\text { Baseline } \\
1 \text { year } \\
2 \text { years } \\
5 \text { years }\end{array}$ \\
\hline Zhang, 2017 & $\begin{array}{l}\text { Baseline } \\
3 \text { months } \\
7 \text { months } \\
11 \text { months } \\
15 \text { months }\end{array}$ \\
\hline Reme, 2019 & $\begin{array}{l}\text { Baseline } \\
6 \text { months } \\
12 \text { months } \\
18 \text { months }\end{array}$ \\
\hline
\end{tabular}

Studies of people with organic mental disorder, learning disorder or minor psychiatric disorders, first psychotic episodes, case studies and specific sample of people over 65 years of age were excluded.

Once the studies that met the inclusion criteria had been identified, the contents were compiled into data extraction sheets previously designed by the group of reviewers.

Three hundred and eighty-three references were reviewed, of which 26 were selected and 18 randomized clinical studies were included. The information was then classified in tables following a standardized protocol to report the results found in the non-vocational variables.

\section{Results}

Of the 26 selected RCTs, 18 studies met the inclusion criteria and were included. Eight studies were excluded because they did not present clear non-vocational outcomes, or were specific to first episodes of psychosis, elderly people, post-traumatic stress or criminals in prison.

Employment interventions are generally divided into two large groups, with the IPS strategy predominating over the Traditional Vocational Rehabilitation (TVR) strategy. There are two studies that add the IPS strategy combined with others such as Assertive Community Treatment (ACT) [28] and Work-related Social Skill Training (WSST) [26], the latter combination giving rise to Integrated Supported Employment (ISE) (Table 1).

Studies have shown that the follow-up period (Table 2) varies from 12 months [10], 15 months [26], 18 months [20,21,23,24,27,30-32,36], 24 months $[22,25,28,29,33,34]$ and 5 years [35].

Sample sizes vary according to the number of participants (Table 1). Most commonly, the sample is between 100 and 200 persons $[10,20,21,26,28,29,34-36]$. Less than 100 people were found in only two studies [25,31] and seven studies found large samples, larger than 200 people [22-24,27,30,32,33].

Regarding results, eight studies [22-24,28,29,32,34,35] used the Positive and Negative Syndrome Scale (PANSS), two trials [24,27] used the Hospital Anxiety and Depression Scale (HADS), and seven studies $[10,20,21,25,26,31,36]$ used the Brief Psychiatric Rating Scale (BPRS).

\section{Symptomatology and employment}

On the analysis of symptoms, seven of the analyzed RCTs reported significant changes and results in the symptomatology variable in favor of the competitive employment group with the IPS strategy [21,22,2427,32]. The group working on competitive employment with the IPS strategy is shown to improve in symptoms, overall functioning and well-being. In addition, one of the studies found that having a job reduced the risk of hospital admissions [32].

Bond et al. 2001 [21], found that the competitive work group showed higher rates of improvement in symptoms (BPRS symptoms, $f(432)=-2.17, p=0.031, E S=0.70$; as well as for the subscales of Affection, $\mathrm{f}(433)=-2.16, \mathrm{p}=0.032$, ES $=0.48$, and Disorganization, $\mathrm{f}(433)=-1.99$, $\mathrm{p}=0.047, \mathrm{ES}=0.54$ ), in satisfaction with vocational services, leisure and finances, as well as improvement in self-esteem. The protected or unemployed work group did not show this advantage.

In the 2004 study by Mueser et al. [22] they found an improvement in both symptoms and overall functioning (GAS and PANSS). They found effects that were significant, although the value of $\mathrm{p}$ is not specified (standard services 2.3 (.8) vs IPS 2.3 (.9) vs PSR 2.4 (.8)). 
Pulido FR (2020) Review of individual placement and support (IPS) studies and results on health status of people with long-term mental disorder and competitive employment

Table 3. Symptomatology results (BPRS: Brief Psychiatric Rating Scale. GAF: Global Assessment of Functioning. GAS: Global Assessment Scale. HADS: Hospital Anxiety and Depression Scale. PANSS Positive and Negative Syndrome Scale. WHODAS: World Health Organization Disability Assessment Schedule)

\begin{tabular}{|c|c|c|}
\hline Study & Symptomatology & Results \\
\hline \multirow{2}{*}{ Drake, 1999} & \multirow{2}{*}{ Yes } & $\begin{array}{c}\text { GAS, M (SE) } \\
\text { IPS } \\
\text { Baseline } 42.7(1.08) \\
6 \text { months } 44.2(1.17) \\
12 \text { months } 46.2(1.32) \\
18 \text { months } 45.8(1.43) \\
\text { EVR } \\
\text { Baseline } 42.2(1.11) \\
6 \text { months } 44.1(1.15) \\
12 \text { months } 45.1(1.16) \\
18 \text { months } 46.0(1.78)\end{array}$ \\
\hline & & $\begin{array}{c}\text { BPRS total, M (SE) } \\
\text { IPS } \\
\text { Baseline } 37.4(1.01) \\
6 \text { months } 38.3(1.33) \\
12 \text { months } 38.5(1.16) \\
18 \text { months } 39.2(1.19) \\
\text { EVR } \\
\text { Baseline } 37.6(1.11) \\
6 \text { months } 39.6(1.31) \\
12 \text { months } 39.0(1.32) \\
18 \text { months } 41.1(1.54)\end{array}$ \\
\hline \multirow[b]{2}{*}{ Bond, 2001} & \multirow[b]{2}{*}{ Yes } & $\begin{array}{c}G A S, \mathrm{M} \\
\text { Competitive work } 47.2 \\
\text { Sheltered work } 39.6 \\
\text { Minimal work } 41.0 \\
\mathrm{f}(3,148)=3.87, \mathrm{p}=0.011\end{array}$ \\
\hline & & $\begin{array}{c}\text { BPRS total } \\
\mathrm{f}(432)=-2.17, \mathrm{p}=0.031, \mathrm{ES}=0.70 \\
\text { Affection } \\
\mathrm{f}(433)=-2.16, \mathrm{p}=.032, \mathrm{ES}=0.48 \\
\text { Disorganization } \\
\mathrm{f}(433)=-1.99, \mathrm{p}=0.047, \mathrm{ES}=0.54\end{array}$ \\
\hline & & $\begin{array}{c}G A S, \mathrm{M} \\
\text { Baseline } 50.9 \\
2 \text { years } 53.8\end{array}$ \\
\hline Mueser, 2004 & Yes & $\begin{array}{c}\text { PANSS factors, M (SD) } \\
\text { Baseline } \\
\text { Negative } \\
\text { Standard service 2.3 (.9) } \\
\text { IPS } 2.3(1.0) \\
\text { PSR } 2.4(.8) \\
\text { Positive } \\
\text { Standard service } 2.2(1.0) \\
\text { IPS } 1.8(.8) \\
\text { PSR } 2.0(.8) \\
\text { Excitement } \\
\text { Standard service } 1.7(.8) \\
\text { IPS } 1.5(.5) \\
\text { PSR } 1.7(.7) \\
\text { Depression } \\
\text { Standard service 2.0 (.9) } \\
\text { IPS } 1.9(.7) \\
\text { PSR 2.1 }(.8) \\
\text { Cognitive } \\
\text { Standard service 2.3 (.8) } \\
\text { IPS } 2.3(.9) \\
\text { PSR 2.4 (.8) }\end{array}$ \\
\hline
\end{tabular}


Pulido FR (2020) Review of individual placement and support (IPS) studies and results on health status of people with long-term mental disorder and competitive employment

\begin{tabular}{|c|c|c|}
\hline Gold, 2006 & Yes & $\begin{array}{c}\text { PANSS items, } \mathrm{M}(\mathrm{SD}) \\
\text { Positive } \\
\text { SE } 7.9(3.2) \\
\text { ACT }+\mathrm{IPS} 8.3(3.4) \\
\mathrm{t}(141)=-0.72, \mathrm{p}=.47 \\
\text { Negative } \\
\text { SE } 17.0(6.0) \\
\text { ACT-IPS 16.9 (5.1) } \\
\mathrm{t}(141)=0.08, \mathrm{p}=.93 \\
\text { Autistic preoccupation } \\
\text { SE } 11.2(3.8) \\
\text { ACT-IPS } 11.4(4.0) \\
\mathrm{t}(141)=-0.24, \mathrm{p}=.81 \\
\text { Activation } \\
\text { SE } 7.1(2.6) \\
\text { ACT-IPS } 7.6(3.3) \\
\mathrm{t}(141)=-1.10, \mathrm{p}=.27 \\
\text { Dysphoria } \\
\text { SE } 12.5(4.6) \\
\text { ACT-IPS } 13.1(5.2) \\
\mathrm{t}(141)=-0.67, \mathrm{p}=.51\end{array}$ \\
\hline \multirow{2}{*}{ Latimer, 2006} & \multirow{2}{*}{ Yes } & $\begin{array}{c}\text { GAF, M (SD) } \\
\text { Baseline } \\
\text { US } 58.6(12.9) \\
\text { SE } 62.0(12.4) \\
\text { p }=0.14\end{array}$ \\
\hline & & $\begin{array}{l}\text { BPRS global, M (SD) } \\
\text { Baseline } \\
\text { US } 43.3(15.5) \\
\text { SE } 41.63(12.3) \\
\text { p }=0.74\end{array}$ \\
\hline Bond, 2007 & Yes & $\begin{array}{l}\text { PANSS, M (SD) } \\
\text { Baseline } \\
\text { IPS } 1.97(0.46) \\
\text { DPA } 2.05(0.53) \\
\mathrm{t}=-1.07, \mathrm{p}=.29\end{array}$ \\
\hline Burns, 2007 & No & - \\
\hline Burns, 2008 & Yes & $\begin{array}{l}\text { GAS/F } \\
\text { PANSS }\end{array}$ \\
\hline Wong, 2008 & Yes & $\begin{array}{c}B P R S \\
\text { Vocacional vs No vocacional } \\
\begin{array}{c}\text { There are no differences between the two groups, since there are no substantial changes in } \\
\text { psychiatric symptoms. }\end{array}\end{array}$ \\
\hline & & $\begin{array}{c}G A F-S, \text { M (SD) / D }(95 \% \mathrm{CI}) \\
\text { IPS 55.5 }(11.94) \\
\text { VS } 55.3(13.04) \\
\text { Total } 55.4(12.48) \\
G A F-D, \text { M (SD) / D (95\% CI) } \\
\text { IPS } 53.9(12.93) \\
\text { VS } 53.7(13.38) \\
\text { Total } 54.1(13.72)\end{array}$ \\
\hline Burns, 2009 & Yes & $\begin{array}{c}\text { PANSS, M (SD) / D }(95 \% \mathrm{CI}) \\
\text { Baseline } \\
\text { Positive } \\
\text { IPS } 13.3(4.85) \\
\text { VS } 13.4(5.39) \\
\text { Total } 13.4(5.12) \\
\text { Negative } \\
\text { IPS } 14.7(6.20) \\
\text { VS } 15.3(6.31) \\
\text { Total } 15.0(6.25) \\
\text { General } \\
\text { IPS } 31.3(8.67) \\
\text { VS } 31.3(8.95) \\
\text { Total } 31.3(8.80) \\
18 \text { months } \\
\text { Positive } \\
\text { IPS } 12.7(4.84) \\
\text { VS } 12.6(4.40) \\
0.028(-0.928 \text { to } 0.983) \\
\text { Negative } \\
\text { IPS } 13.3(5.13) \\
\text { VS } 13.5(5.47) \\
0.084(-0.983 \text { to } 1.15) \\
\text { General } \\
\text { IPS } 29.3(7.82) \\
\text { VS } 28.9(7.87) \\
0.455(-1.11 \text { to } 2.02)\end{array}$ \\
\hline
\end{tabular}


Pulido FR (2020) Review of individual placement and support (IPS) studies and results on health status of people with long-term mental disorder and competitive employment

\begin{tabular}{|c|c|c|}
\hline & & $\begin{array}{c}\text { HADS, M (SD) / D }(95 \% \mathrm{CI}) \\
\text { Baseline } \\
\text { Anxiety } \\
\text { IPS } 7.1(4.46) \\
\text { VS } 6.5(4.60) \\
\text { Total } 6.8(4.53) \\
\text { Depression } \\
\text { IPS } 6.6(4.08) \\
\text { VS } 5.8(4.24) \\
\text { Total } 6.2(4.17) \\
18 \text { months } \\
\text { Anxiety } \\
\text { IPS } 6.5(4.53) \\
\text { VS } 6.4(4.30) \\
-0.221(-1.07 \text { to } 0.632) \\
\text { Depression } \\
\text { IPS } 6.1(4.24) \\
\text { VS } 6.2(4.56) \\
-0.302(-1.21 \text { to } 0.606)\end{array}$ \\
\hline \multirow{2}{*}{ Nygren, 2011} & \multirow{2}{*}{ Yes } & $\begin{array}{c}\text { GAF, M [IR] } \\
\text { Baseline } \\
\text { Employment 62 (64) [55-68 (60-68)] } \\
\text { Education 62 (68) [59-65 (65-70] } \\
\text { Work practice 57 (61) [53-65(55-65)] } \\
\text { No occupation 56(57) [53-60(54-63)] }\end{array}$ \\
\hline & & $\begin{array}{c}B P R S, \mathrm{M}[\mathrm{IR}] \\
\text { Baseline } \\
\text { Employment } 29(26)[27-32(23-32)] \\
\text { Education 28 (25) [25-30(23-29)] } \\
\text { Work practice 33(29) [29-37(27-30)] } \\
\text { No occupation 31 (30) [28-34 (27-33)] }\end{array}$ \\
\hline Kilian, 2012 & Yes & $\begin{array}{c}\text { PANSS, M (SD) } \\
\text { Baseline 59.64 (16.97) } \\
6 \text { months 57.15 (17.08) } \\
12 \text { months 56.02 (16.45) } \\
18 \text { months 55.09 }(14.92)\end{array}$ \\
\hline Drake, 2013 & No & - \\
\hline Kukla, 2013 & Yes & $\begin{array}{c}\text { PANSS total, M (SD) } \\
\text { Baseline } \\
\text { IPS } 59.24(13.84) \\
\text { DPA } 61.56(15.83) \\
6 \text { months } \\
\text { IPS } 58.15(16.61) \\
\text { DPA } 61.56(16.11) \\
12 \text { months } \\
\text { IPS } 52.00(25.14) \\
\text { DPA } 51.16(28.64) \\
18 \text { months } \\
\text { IPS } 50.07(28.30) \\
\text { DPA 47.32 (31.98) } \\
24 \text { months } \\
\text { IPS } 59.90(15.97) \\
\text { DPA } 62.95(19.16)\end{array}$ \\
\hline Bejerlhom, 2014 & $\mathrm{Si}$ & $\begin{array}{c}\text { BPRS total, M (SD) } \\
\text { Baseline } \\
\text { IPS 1.44 (0.37) } \\
\text { TVR 1.49 (0.34) } \\
\mathrm{Z}=-0.295, \mathrm{p}=0.768\end{array}$ \\
\hline \multirow[t]{2}{*}{ Hoffman, 2014} & \multirow[t]{2}{*}{ Yes } & $\begin{array}{l}\text { PANSS, M (SD) } \\
\text { Baseline } \\
\text { Positive } \\
\text { SEP } 9.5(3.4) \\
\text { TVR } 8.6(2.3) \\
\text { Negative } \\
\text { SEP 11.2 (4.7) } \\
\text { TVR } 10.3(3.3) \\
\text { General } \\
\text { SEP } 25.4(7.4) \\
\text { TVR } 23.1(5.1)\end{array}$ \\
\hline & & $\begin{array}{c}G A F, \mathrm{M}(\mathrm{SD}) \\
\text { Baseline } \\
\text { SEP } 49.8(6.6) \\
\text { TVR } 49.9(5.5)\end{array}$ \\
\hline
\end{tabular}


Pulido FR (2020) Review of individual placement and support (IPS) studies and results on health status of people with long-term mental disorder and competitive employment

\begin{tabular}{|c|c|c|}
\hline \multirow{2}{*}{ Zhang, 2017} & \multirow{2}{*}{ Yes } & $\begin{array}{c}G A F, \mathrm{M}(\mathrm{SD}) \\
7 \text { months } \\
\text { ISE } 64.91(3.83) \\
\text { IPS } 63.00(1.64) \\
\text { TVR } 62.15(1.94) \\
11 \text { months } \\
\text { ISE } 65.00(3.88) \\
\text { IPS } 63.50(2.04) \\
\text { TVR } 62.39(2.06)\end{array}$ \\
\hline & & $\begin{array}{c}B P R S, \mathrm{M}(\mathrm{SD}) \\
7 \text { months } \\
\text { ISE } 24.41(2.27) \\
\text { IPS } 24.59(2.02) \\
\text { TVR } 24.43(1.81) \\
11 \text { months } \\
\text { ISE } 24.41(2.24) \\
\text { IPS } 24.07(1.91) \\
\text { TVR } 24.07(1.76)\end{array}$ \\
\hline \multirow{2}{*}{ Reme, 2019} & \multirow{2}{*}{ Yes } & $\begin{array}{c}\text { WHODAS, M }(95 \% \mathrm{CI}) / \mathrm{D}(95 \% \mathrm{CI}) \text { ES }(95 \% \mathrm{CI}) \\
\text { Baseline } \\
\text { IPS } 22.47(20.48-24.46) \\
\text { VR } 23.48(21.14-25.82) \\
6 \text { months } \\
\text { IPS } 22.57(19.68-25.46) \\
\text { VR } 24.20(20.69-27.70) \\
-1.63(-6.03-2.78) \\
\mathrm{p}=0.469 \\
-0.10(-0.38-0.17) \\
12 \text { months } \\
\text { IPS } 19.60(16.81-22.4) \\
\text { VR } 28.70(25.34-32.05) \\
-9.09(-13.3--4.87) \\
\mathrm{p}<0.001 \\
-0.57(-0.83--0.30)\end{array}$ \\
\hline & & $\begin{array}{c}\text { HADS, M }(95 \% \mathrm{CI}) / \mathrm{D}(95 \% \mathrm{CI}) \mathrm{ES}(95 \% \mathrm{CI}) \\
\text { Baseline } \\
\text { IPS } 15.97(14.97-16.97) \\
\text { VR } 16.13(15.00-17.25) \\
6 \text { months } \\
\text { IPS } 20.64(19.28-22.01) \\
\text { VR } 20.28(18.66-21.91) \\
0.36(-1.75-2.47) \\
\mathrm{p}=0.737 \\
0.05(-0.24-0.34) \\
12 \text { months } \\
\text { IPS } 13.74(12.38-15.09) \\
\text { VR } 16.74(15.09-18.39) \\
-3.00(-5.13--0.87) \\
\mathrm{p}=0.006 \\
-0.41(-0.70--0.12)\end{array}$ \\
\hline
\end{tabular}

Table 4. Hospitalization outcomes

\begin{tabular}{|c|c|c|}
\hline Study & Hospitalization & Results \\
\hline & & $\begin{array}{c}\text { Days in hospital, M (SE) } \\
\text { IPS } 30.3(6.5) \\
\text { EVR } 17.4(3.9)\end{array}$ \\
\hline Drake, 1999 & Yes & $\begin{array}{c}\text { Hospital days, M (SE) } \\
\text { IPS } \\
\text { Baseline } 15.2(3.24) \\
\frac{6 \text { months }}{12 \text { months }} 10.0(2.39) \\
\frac{\underline{18 \text { months }}}{\text { EVR }} 11.5(2.43) \\
\text { Baseline } 8.7(1.96) \\
\underline{\text { 6 months }} 7.7(1.79) \\
\underline{12 \text { months }} 4.7(1.32) \\
\underline{18 \text { months }} 5.7(1.33)\end{array}$ \\
\hline Bond, 2001 & Yes & $\begin{array}{c}\text { Days in hospital during the previous year, } \mathrm{M}(\mathrm{SD}) \\
\text { Competitive work } 16.5(27.8) \\
\text { Sheltered work } 23.8(44.5) \\
\text { Minimal work } 28.6(62.8) \\
\text { No work } 22.9(32.6)\end{array}$ \\
\hline
\end{tabular}


Pulido FR (2020) Review of individual placement and support (IPS) studies and results on health status of people with long-term mental disorder and competitive employment

\begin{tabular}{|c|c|c|}
\hline Mueser, 2004 & Yes & $\begin{array}{c}\text { Months lifetime psychiatric hospitalization, M (SD) } \\
\text { Baseline } \\
\text { Standard service } 15.3(22.2) \\
\text { IPS } 18.8(30.6) \\
\text { PSR } 21.7(52.3)\end{array}$ \\
\hline \multirow{2}{*}{ Gold, 2006} & \multirow{2}{*}{ Yes } & $\begin{array}{c}\text { Partial hospitalization, N (\%) } \\
\text { SE 2 (3) } \\
\text { ACT+IPS 1 (2) }\end{array}$ \\
\hline & & $\begin{array}{c}\text { Psychiatric hospitalization, N (\%) } \\
\text { SE } 21(27) \\
\text { ACT+IPS } 17 \text { (26) }\end{array}$ \\
\hline Latimer, 2006 & No & - \\
\hline Bond, 2007 & Yes & $\begin{array}{c}\text { Days in hospital prior year, M (SD) } \\
\text { Baseline } \\
\text { IPS } 15.54(25.37) \\
\text { DPA } 14.73(26.20) \\
\mathrm{t}=0.22, \mathrm{p}=.83\end{array}$ \\
\hline Burns, 2007 & Yes & $\begin{array}{c}\text { Percentage of time spent in hospital, } \mathrm{M}(\mathrm{SD}) / \mathrm{D}(95 \% \mathrm{CI}) \\
\text { IPS } 4.6(13.56) \\
\text { VS } 8.9(20.08) \\
-4.3(-8.40 \text { to }-0.59)\end{array}$ \\
\hline \multirow{2}{*}{ Burns, 2008} & \multirow{2}{*}{ Yes } & $\begin{array}{c}\text { Hospitalized, } \mathrm{N}(\%) / \mathrm{D}(95 \% \mathrm{CI}) \\
\text { IPS } 28(20.1) \\
\text { VS } 42(31.3) \\
-11.2(-21.5,-0.90)\end{array}$ \\
\hline & & $\begin{array}{c}\text { Percentage of time spent in hospital, M (SD) / D }(95 \% \mathrm{CI}) \\
\text { IPS } 4.6(13.56) \\
\text { VS } 8.9(20.08) \\
-4.3(-8.40,-0.59)\end{array}$ \\
\hline \multirow{2}{*}{ Wong, 2008} & \multirow{2}{*}{ Yes } & $\begin{array}{l}\text { Number of previous psychiatric hospitalizations in the past } 2 \text { years, } \mathrm{M} \pm \mathrm{SD} \\
\text { Baseline } \\
\text { SE } 1.4 \pm 1.2 \\
\text { CVR } 1.7 \pm 1.5\end{array}$ \\
\hline & & $\begin{array}{l}\text { Days in a psychiatric hospital in the past } 2 \text { years, } \mathrm{M} \pm \mathrm{SD} \\
\text { Baseline } \\
\text { SE } 161 \pm 190 \\
\text { CVR } 171 \pm 187\end{array}$ \\
\hline \multirow[t]{2}{*}{ Burns, 2009} & \multirow[t]{2}{*}{ Yes } & $\begin{array}{l}\text { Hospitalized in final } 6 \text { months, } \mathrm{N}(\%) / \mathrm{D}(95 \% \mathrm{CI}) \\
\qquad \begin{array}{c}18 \text { months } \\
\text { Subscale Yes } \\
\text { IPS } 11(8.3) \\
\text { VS 22 }(18.3) \\
\text { Subscale No } \\
\text { IPS 121 (91.7) } \\
\text { VS } 98(81.7) \\
2.16(0.972 \text { to } 4.79)\end{array}\end{array}$ \\
\hline & & $\begin{array}{c}\text { Hospitalized in previous } 6 \text { months, } \mathrm{D}(95 \% \mathrm{CI}) \\
\text { Subscale Yes } \\
\text { Total } 0.352(0.115 \text { to } 0.818) \\
\text { IPS } 0.347(0.116 \text { to } 1.03) \\
\text { VS } 0.633(0.164 \text { to } 2.45)\end{array}$ \\
\hline Nygren, 2011 & No & - \\
\hline Kilian, 2012 & Yes & $\begin{array}{c}\text { Days in hospital during the past } 6 \text { months, } \mathrm{M}(\mathrm{SD}) \\
\text { Baseline } 0 \\
\underline{6 \text { months } 7.20(21.61)} \\
\underline{12 \text { months }} 7.07(20.79) \\
\underline{18 \text { months }} 5.79(18.50)\end{array}$ \\
\hline \multirow{4}{*}{ Drake, 2013} & \multirow{4}{*}{ Yes } & $\begin{array}{l}\text { Overnight hospital stays, } \mathrm{M}(\mathrm{SD}) \\
\text { IPS } 0.86(1.67) \\
\text { SAU } 0.97(1.76) \\
\mathrm{MD}=-0.12, \mathrm{p}=0.092, \mathrm{~d}=-0.06\end{array}$ \\
\hline & & $\begin{array}{l}\text { Overnight hospital days, M (SD) } \\
\text { IPS } 5.74(16.31) \\
\text { SAU } 7.37(18.96) \\
\text { MD }=-1.63, \mathrm{p}=0.008, \mathrm{~d}=-0.09\end{array}$ \\
\hline & & $\begin{array}{c}\text { Overnight mental health hospital stays, } \mathrm{M}(\mathrm{SD}) \\
\text { IPS } 0.40(1.20) \\
\text { SAU } 0.45(1.17) \\
\mathrm{MD}=-0.05, \mathrm{p} 0.316, \mathrm{~d}=-0.04\end{array}$ \\
\hline & & $\begin{array}{c}\text { Overnight mental health hospital days, } \mathrm{M}(\mathrm{SD}) \\
\text { IPS } 3.86(14.22) \\
\text { SAU } 4.92(16.80) \\
\mathrm{MD}=-1.05, \mathrm{p}=0.101, \mathrm{~d}=-0.07\end{array}$ \\
\hline
\end{tabular}


Pulido FR (2020) Review of individual placement and support (IPS) studies and results on health status of people with long-term mental disorder and competitive employment

\begin{tabular}{|c|c|c|}
\hline \multirow{3}{*}{ Kukla, 2013} & \multirow{3}{*}{ Yes } & $\begin{array}{l}\text { Hospitalizations year prior, } \mathrm{M}(\mathrm{SD}) \\
\text { IPS } 14.90(25.39) \\
\text { DPA } 13.74(25.97) \\
\text { MW=-1.34, } \mathrm{p}=0.18\end{array}$ \\
\hline & & $\begin{array}{l}\text { Hospitalizations year one, } \mathrm{M}(\mathrm{SD}) \\
\text { IPS } 4.86(12.71) \\
\text { DPA } 8.32(22.60) \\
\mathrm{MW}=0.42, \mathrm{p}=0.67\end{array}$ \\
\hline & & $\begin{array}{l}\text { Hospitalizations year two, M (SD) } \\
\text { IPS 5.59 (13.31) } \\
\text { DPA } 9.69(24.24) \\
\text { MW }=0.67, \mathrm{p}=0.50\end{array}$ \\
\hline Bejerlhom, 2014 & Yes & $\begin{array}{c}\text { Hospital admissions, M (SD) } \\
\text { Baseline }(\mathrm{n}=111) \\
\text { IPS } 3.12(3.7) \\
\text { TVR } 4.09(7.1) \\
\text { t(109) }=-0.917, \mathrm{p}=0.361\end{array}$ \\
\hline \multirow{2}{*}{ Hoffman, 2014} & \multirow{2}{*}{ Yes } & $\begin{array}{l}\text { Number of previous psychiatric hospitalizations, M (SD) } \\
\text { Baseline } \\
\text { SEP } 1.8(2.8) \\
\text { TVR } 1.6(2.2)\end{array}$ \\
\hline & & $\begin{array}{c}\text { Months in hospital, M (SD) } \\
\text { Baseline } \\
\text { SEP } 5.5(6.7) \\
\text { TVR } 4.0(4.8)\end{array}$ \\
\hline Zhang, 2017 & No & - \\
\hline Reme, 2019 & No & - \\
\hline
\end{tabular}

In 2009 Burns et al. [24] concluded that the work was associated with improved functioning and fewer symptoms. When they analysed total duration of work ("days worked") as a variable, there were small but significant associations between days worked and overall functioning in terms of symptoms and disability, negative symptoms, general symptoms and social disability. Working 90 more days was associated with better overall functioning in terms of symptoms (by 1.8 points out of 100 in GAF-S), better overall functioning in terms of disability (by 2.7 points out of 100 in GAF-D), fewer negative symptoms (by 0.9 points out of 42 in PANSS), fewer general symptoms (by 0.9 points out of 42 in PANSS), and less social disability (by 0.9 points out of 21 in GSDS). Starting work was associated with better overall functioning in terms of symptoms and disability, with fewer overall symptoms, at 1.6 points lower ( $95 \% \mathrm{CI}=0.48$ to 2.74$)$.

In the 2011 trial by Nygren et al. [25], employed participants showed improvements in psychiatric symptoms at 12 months $(\mathrm{p}=0.000)$ and in overall functioning $(\mathrm{p}=0.002)$.

In addition, Kilian et al. in 2012 [32] demonstrate that having a competitive job has a positive influence on the level of psychopathology of people with schizophrenia and reduces the risk of hospital admissions (PANSS total score Intercept Mean 59.06 (0.000) Variance 258.64 (0.005) Slope Mean -1.44 (0.132) Variance 12.64 (0.000)).

In 2017, Zhang et al. [26] found that participants with IPS plus WSST (ISE strategy) significantly improved psychiatric symptoms, social functioning and well-being (BPRS ISE: 23.11 (2.66) IPS: 23.46 (1.99) TVR 23.87 (1.71) Time Repeated Measures ANOVA 6.36 0.00).

In Reme et al. in 2019 [27], the IPS group produced positive effects on overall functioning, quality of life and symptoms (Total HADS (042) $\mathrm{p}=0.006$; WHODAS Disability $(0-100) \mathrm{p}<0.001$; Health-related QoL (0-100) p=0.008; Global well-being (1-10) p=0.012).

On the other hand, when analyzing this variable of symptomatology we also observed that in six of the analyzed RCTs they found no significant differences between groups or they do not show significant results $[10,20,28,29,34,36]$. Therefore, both the control and the treatment groups did not obtain improvements or substantial changes in this variable.

In the 1999 study by Drake et al [20], total BPRS scores increased significantly, but these increases were clearly in the subclinical range, with no average symptom scores above 2.0 and no increases in the subscales.

In Gold et al. 2006 [28] no substantial differences were found between the two programs and there was no change from baseline for psychiatric symptoms using the PANSS scale ( $\mathrm{p}=0.27$ for PANSSactivation subscale to $\mathrm{p}=0.93$ for PANSS-negative).

Latimer et al. in 2006 [10] found no significant difference in symptoms with the BPRS scale (US (mean (SD) 43.3 (15.5) vs IPS (mean (SD) 41.3 (12.3), $\mathrm{p}=0.74$ ).

In the 2007 Bond et al. trial [29], with the PANSS scale, no significant results were obtained between both groups (PANSS IPS 1.97 (0.46) vs DPA 2.05 (0.53), $\mathrm{t}=-1.07, \mathrm{p}=0.29)$.

In 2013, according to Kukla and Bond [34], both groups reported mild to moderate symptom levels throughout the study period without significant results (total PANSS (IPS vs DPA); baseline 59.24 (13.84) vs 61.56 (15.83); 24 months 59.90 (15.97) vs 62.95 (19.16); $\mathrm{df}=1,182.3$, $\mathrm{F}=1.40, \mathrm{p}=0.24)$.

And in Bejerlhom et al. in 2015 [36] they also obtained no change in symptoms using the BPRS scale (total BPRS IPS $=1.44(0.37)$ vs TVR=1.49 (0.34) $\mathrm{Z}=-0.295 ; \mathrm{p}=0.768)$.

\section{Hospitalizations and employment}

In six of the studies, the IPS group was found to have significantly fewer hospitalizations or relapses than the control group $[23,24,30,32,33,35]$. Three studies had fewer hospitalizations or hospital admissions over the follow-up time, but this was not found to be significant or the significance was not specified [20,34,36], and three others looked at pre-employment hospitalization [21,29,31]. One study [28] shows that both study groups had hospitalizations without 
Pulido FR (2020) Review of individual placement and support (IPS) studies and results on health status of people with long-term mental disorder and competitive employment

a significance level (IPS+ACT: $17.26 \%$ and SE: $21.27 \%)$ and one found more months of psychiatric hospitalization at baseline [22].

The study by Burns et al. in 2007 [30] concluded that the traditional service was significantly more likely to have twice the average time in hospital and in the study by Burns et al. of 2008 [23] they were significantly less likely to have relapses or rehospitalizations. In addition, one of the unexpected findings was the variable hospitalization that was reduced in patients who received IPS (time spent in hospital: IPS 4.6 (13.56) vs VS 8.9 (20.08), difference $-4.395 \%$ CI (-8.40, -0.59)). In the trial conducted by Burns et al. in 2009 [24], not working was found to be associated with a higher likelihood of hospitalization. Patients who did not work were 2.84 times more likely to be hospitalized in the last 6 months $(95 \% \mathrm{CI}=1.22,8.70)$ and 1.98 times more likely to be in remission during the previous 6 months $(95 \% \mathrm{CI}=1.15,3.40)$.

In 2012, Kilian et al. [32] found that having a job reduced the risk of hospital admissions and related hospitalization days to the PANSS scale. They found that the number of days of hospitalization during each time interval was positively related to the PANSS score at the beginning of the interval ( $\mathrm{b} 0.201 ; \mathrm{p}=0.020)$ and to the number of days of hospitalization during the previous interval ( $\mathrm{b} 0.502 ; \mathrm{p}=0.000$ ).

In Drake et al. in 2013 [33] there were significant differences in days of hospital stay in favour of IPS and in Hoffman et al. in 2014 [35] participants in the IPS group had significantly fewer days of hospitalization.

The study by Drake et al. in 1999 [20] found that admissions were reduced after starting the vocational program although the reductions were not significant. Both study groups decreased hospital admissions after starting their vocational programs even though the IPS group obtained a significantly higher number of days of hospitalization.

In the 2013 study by Kukla et al. [34] there was a finding that reflected that working long periods of time in competitive jobs may be the key ingredient associated with improvements in non-vocational fields over time. Both groups in this study reduced their admissions after two years of follow-up.

The 2014 study by Bejerhom et al. [36] found that the IPS group obtained fewer hospital admissions than the TVR group but without being significant (IPS 3.12 (3.7) vs TVR 4.09 (7.1), statistical test $(\mathrm{t}(109)=-0.917 ; \mathrm{p}=0.361)$.

In three of the trials $[21,29,31]$ hospitalizations were analyzed prior to entering the employment program without finding significant differences between the groups and were not analyzed during the follow up of the employment programs.

In 2001, Bond et al. [21] measured the days spent in hospital during the previous year and the competitive IPS working group obtained the fewest days spent in hospital (the study lacked significance).

In the 2007 Bond et al. trial [29], psychiatric hospitalization admissions and days hospitalized were measured for the year prior to enrollment based on information from participant self-report, hospital discharge summaries, and Medicaid claims with a non-significant difference.

Wong et al. in 2008 [31] found no difference in days of hospitalization or number of psychiatric hospitalizations in the previous 2 years.

In 2006, Gold et al. [28] found that the two study groups had hospitalizations. The Supported Employment Program (SEP) had
$21.27 \%$ and the ACT with IPS (ACT+IPS) had $17.26 \%$ but did not specify the value of significance (p).

In the 2004 study by Mueser et al. [22], months of hospitalization were higher in the IPS group at baseline than in the standard services (standard services 15.3 (22.2) vs IPS 18.8 (30.6) vs PSR 21.7 (52.3)).

\section{Discussion}

The psychological and social benefits of work for people with mental health problems is a conscious result of all research, but the benefits that work itself creates for people's lives are very diverse.

In this review, we have deepened in the results using the IPS strategy and the symptomatology and hospitalization variables they have for people who get a job.

Fourteen of the RCT studies analyzed studied psychiatric hospitalizations/hospital admissions [20-24,28-36]. Six of these studies found that the IPS group had significantly fewer relapses and hospitalizations compared to the control group, three studies showed fewer hospitalizations in the IPS group without being significant, three of the studies were analyzed prior to employment but did not analyze hospitalizations during follow up after entering employment programs, and only two studies found worse outcomes in the IPS group but it should be noted that one of these does not show significant outcomes [28] and one refers to the study baseline [22]. These results show that no studies have been found that significantly confirm the hypothesis that having a competitive job increases stress and the risk of hospitalization.

However, there are studies that do seem to significantly tip the balance that employment can reduce the risk of hospitalization. The Burns et al. studies of 2008 [23] and 2009 [24] concluded that being in an employment program with IPS methodology was significantly related to being less likely to have relapses or rehospitalizations. Another conclusive study is that of Kilian et al. in 2012 [32] which reflects that having a job reduced the risk of hospital admissions. All models indicated that patients who received an intervention with the IPS model also spent more hours in competitive employment and, because of the positive indirect effects of employment on clinical status, spent fewer days in psychiatric hospitals than patients who received conventional vocational training [32]. These data are consistent with the results found in a European study [6] where the mean annual hospital admissions in the IPS group was 0.19 (SD: 0.548), while the mean in the control group was 2.12 (SD: 2.046), with a difference between the means of $-1.93(95 \%$ CI: -2.41 to -1.45 ). In the IPS group, $86 \%$ of people had no admissions in the follow-up period, compared to $20 \%$ in the control group.

As for the symptomatology variable, one of the problems with the analysis is that it is measured with different scales, although in most studies the PANSS scale or the BPRS scale is used. Seven of the analyzed RCT studies reported significant changes and results in the symptomatology variable in favor of the competitive employment group with the IPS strategy [20-22,24-27] and six found no significant difference in improvement $[10,28,29,31,34,36]$.

When comparing the different studies we found that a significant aspect is that studies with large samples (more than 200 people) have significant results in favor of the IPS in non-vocational outcomes [22$24,27,30,32,33$ ]. It is possible that large sample sizes may better reflect differences from the clinical point of view. However, a Cochrane review [12] including 14 randomised controlled trials (with a total of 2265 people) comparing the IPS strategy with standard psychiatric care found that there was no evidence that supported employment was associated 

employment

with a lower or higher score than other vocational approaches when using the BPRS, PANSS or HADS scales. This review, however, refers to the fact that the IPS strategy can be enhanced with other added interventions such as motivational, social skills training and cognitive rehabilitation [37-41].

Also, a long follow-up period seems to provide added data regarding hospitalizations. One of the longest studies analysed was that of Hoffman et al. [35] with 5 years follow-up, suggesting that long-term employment may be clinically beneficial and provide some protection against relapse and rehospitalisation. These results are not reflected in the short-term follow-up studies. In a 2016 review, Van Rijn et al. [42] found that RCTs were generally conducted in small study populations, and participants were followed for a relatively short period (range 1224 months) with few measurements compared to observational studies (range 1-15 years), making it difficult to determine the impact of work on people's health.

The Cochrane review by Kinoshita et al. [12] concluded that there was no evidence that supported employment was associated with a lower or higher rate of hospital admissions or rehospitalisations than other long-term occupational approaches. In the 2008 Burns et al. study [23], the hospitalization variable was evaluated because of physicians' concerns that a stress component might arise in the IPS sample upon re-entering the labor market and they had no evidence that previous studies had found a reduction in this regard. Finally, their study showed that people in the IPS group reduced hospitalisation. One possible explanation for this is that this may simply reflect a greater integration of health and social services in most of Europe compared to the US. The 2009 study by Burns et al. [24] concluded that patients who are not currently working will be 2.84 times more likely to have been hospitalized in the last 6 months. Being at work was also associated with a reduction in the depression variable. The study clearly demonstrated that there were no clinically detrimental effects of working for the group of patients with severe mental disorder because all the significant associations found favored working patients. The findings suggest that while there may be a direct effect of work on mental health, it is also possible that the association is a consequence of less symptomatic and better functioning patients being helped more successfully to obtain employment. Long-term analysis of the impact of employment on mental health with follow-up after finding employment is therefore important. This idea is related to the one found by Wong et al. in 2008 [31], which, although it found no significant differences between the two groups and no substantial change from the beginning of time for psychiatric symptoms and quality of life, offers a possible explanation regarding the patient profile. The lack of change in psychiatric symptoms in the study may be due in part to the profile of the study participants because most of the participants were outpatients with mild to moderate psychiatric disabilities who manifested very mild residual symptoms that were likely to be stable over time.

We found several limitations in the review to be able to compare all studies and draw firm conclusions. On the one hand, there are those referring to the sample sizes, which differ from one study to another, and on the other hand, the follow-up periods which, although they are usually long, vary from one study to another. Another limitation could be that provided by Burns et al. in 2009 [24] in which only randomization at the potential point of return to work could confirm or refute definitively whether employment, as such, affects clinical and social well-being and such studies are unlikely to be conducted.

One of the limitations of this review relates to the study populations. We have tried to focus on similar study populations of psychotic patients with IPS strategies but it is true that differences between the policies applied in each country may reflect the results found in the studies. For example, in countries with policies that ensure a high level of social protection, the impact of job loss on worker health may be less than in countries with limited social coverage. The 2012 study by McLeod et al. [43] found that the association of unemployment and mortality was much stronger in the United States (low level of social protection) than in Germany (high level of social protection).

Another limitation found is that in most studies non-vocational variables are studied not as primary measures but as secondary outcomes providing more information on the vocational components of employment. Health outcome measures are made in secondary terms of improvement of chronic symptoms and not with intensive analysis of other health variables that could be taken into account (e.g. increase/ decrease of drugs, increases or decreases in mg of medication).

There is also the difficulty of measuring the study variables: the selection of the persons to be studied (with more or less severe symptoms) or the discrimination of the effects and impact on the study variables such as what is due to the intervention group (IPS $v s$. traditional program) or the effect of employment itself.

One aspect that we have not found in the review of the studies is to know what people themselves think about employment, that is, whether or not they are satisfied with the working conditions, colleagues, schedules, etc. We know that positively rated employment is generally associated with a reduction in symptoms, improved disease progression and chances of recovery, and a greater likelihood of avoiding hospitalization and maintaining employment [44]. This idea would be important to take into account for future studies in addition to the opinion of employers themselves.

In this short review we can show that having a competitive job has not had a substantial negative impact on health status in unemployed people with serious mental health problems and studies suggest that long-term employment appears to contribute to being clinically beneficial or at least stabilizing the mental health status of people who manage to maintain it for years.

\section{Conclusions}

We found that the IPS strategy works well in the employment of people with severe mental disorder. The non-vocational results in relation to the number of admissions, days in hospital or symptoms, indicate that the traditional belief that employment for people in recovery means a possible situation of vulnerability and worsening of their psychopathology is not met. In-depth studies on self-esteem and job satisfaction of people with severe mental illness are needed to further analyse this variable. It is also necessary to study in depth the differences between policies that ensure a high level of social protection in comparison to countries with limited social protection and their impact on employability strategies.

\section{References}

1. Crowther R, Marshall M, Bond GR, Huxley P (2001) Vocational rehabilitation for people with severe mental illness. Cochrane Database Syst Rev 2: CD003080.

2. Twamley EW, Jeste DV, Lehman AF (2003) Vocational rehabilitation in schizophre-nia and other psychotic disorders: A literature review and meta-analysis of randomized controlled trials. J Nerv Ment Dis 191: 515-523. [Crossref]

3. Bond GR, Drake RE, Becker DR (2008) An update on randomized controlled trials of evidence-based supported employment. Psychiatr Rehabil J 31: 280-90. [Crossref]

4. Campbell K, Bond GR, Drake RE (2011) Who benefits from supported employment: A meta-analytic study. Schizophr Bull 37: 370-80. [Crossref] 
Pulido FR (2020) Review of individual placement and support (IPS) studies and results on health status of people with long-term mental disorder and competitive employment

5. Rodríguez Pulido F (Ed), Caballero Estebaranz N, González Dávila E, González Lorenzo M, Hernández Álvarez de Sotomayor M, et al. (2011) La autonomía personal en el empleo ordinario de las personas con trastorno mental grave. Canarias: Sinpromi S.L.

6. Rodríguez Pulido F, Caballero Estebaranz N, Tallo Aldana E, Méndez Abad ME, Hernández Álvarez-Sotomayor MC, et al. (2018) Efectividad de una estrategia de apoyo individualizado al empleo para personas con trastornos mentales graves. Gac Sanit 32: 513-518.

7. Rodríguez Pulido F, Caballero Estebaranz N, González Dávila E, Melián Cartaya MJ (2019) Cognitive remediation to improve the vocational outcomes of people with severe mental illness. Neuropsychol Rehabil 1-23. [Crossref]

8. West JC, Wilk JE, Olfson M, Rae DS, Marcus S, et al. (2005) Patterns and quality of treatment for patients with schizophrenia in routine psychiatric practice. Psychiatr Serv 56: $283-291$.

9. Lehman AF, Goldberg R, Dixon LB, McNary S, Postrado L, et al. (2002) Improving employment outcomes for persons with severe mental illnesses. Arch Gen Psychiatry 59: 165-172. [Crossref]

10. Latimer EA, Lecomte T, Becker DR, Drake RE, Duclos I, et al. (2006) Generalisabi-lity of the individual placement and support model of supported employment: Results of a Canadian randomised controlled trial. Br J Psychiatry 189: 65-73. [Crossref]

11. Viering S, Jäger M, Nordt C, Bühler F, Bärtsch B, et al. (2015) Does "individual placement and support" satisfy the users' needs? Front Public Health 3: 1-4. [Crossref]

12. Kinoshita Y, Furukawa TA, Kinoshita K, Honyashiki M, Omori IM, et al. (2013) Supported employment for adults with severe mental illness (review). Cochrane Database Syst Rev.

13. Bailey EL, Ricketts SK, Becker DR, Xie H, Drake RE (1998) Do long-term day treatment clients benefit from supported employment? Psychiatr Rehabil J 22: 24-29.

14. Mueser KT, Becker DR, Wolfe R (2001) Supported employment, job preferences, job tenure and satisfaction. J Ment Health 10: 411-417.

15. Rogers JA (1995) Work is key to recovery. Psychosocial Rehabilitation Journal 18: $5-10$

16. Steele K, Berman C (2001) The day the voices stopped. New York, NY: Basic Books.

17. Clevenger N (2008) In favor of science. Psychiatr Rehabil J31: 277-279.

18. Rodríguez Pulido F (Ed) (2010) La recuperación de las personas con trastorno men-tal grave: Modelo de red de redes: Presente y perspectivas de futuro. Fundación Cana-ria de Salud y Sanidad de Tenerife.

19. Bond GR (2004) Supported employment: Evidence for an evidence-based practice. Psychiatr Rehabil J 27: 347-359.

20. Drake RE, McHugo GJ, Bebout RR, Becker DR, Harris M, et al. (1999) A randomized clinical trial of supported employment for inner-city patients with severe mental disorders. Arch Gen Psychiatry 56: 627-633. [Crossref]

21. Bond GR, Resnick SG, Drake RE, Xie H, McHugo GJ, et al. (2001) Does competiti-ve employment improve nonvocational outcomes for people with severe mental ill-ness? J Consult Clin Psychol 69: 489-501. [Crossref]

22. Mueser KT, Clark RE, Haines M, Drake RE, McHugo GJ, et al. (2004) The Hartford study of supported employment for persons with severe mental illness. $J$ Consult Clin Psychol 72: 479-490.

23. Burns T, White SJ, Catty J; EQOLISE group (2008) Individual placement and sup-port in Europe: the EQOLISE trial. Int Rev Psychiatry 20: 498-502. [Crossref]

24. Burns T, Catty J, White S, Becker T, Koletsi M, et al. (2009) The impact of supported employment and working on clinical and social functioning: Results of an international study of individual placement and support. Schizophr Bull 35: 949-958.

25. Nygren U, Markström U, Svensson B, Hansson L, Sandlund M (2011) Individual placement and support: A model to get employed for people with mental illness - The first Swedish report of outcomes. Scand J Caring Sci 25: 591-598.
26. Zhang GF, Tsui CM, Lu AJB, Yu LB, Tsang HWH, et al. (2017) Integrated supported employment for people with schizophrenia in mainland China: A randomized controlled trial. Am J Occup Ther 71: 7106165020p1-7106165020p8. [Crossref]

27. Reme SE, Monstad K, Fyhn T, Sveinsdottir V, Løvvik C, et al. (2019) A randomized controlled multicenter trial of individual placement and support for patients with moderate-to-severe mental illness. Scand J Work Environ Health 45: 33-41. [Crossref]

28. Gold PB, Meisler N, Santos AB, Carnemolla MA, Williams OH, et al. (2006) Randomized trial of supported employment integrated with assertive community treatment for rural adults with severe mental illness. Schizophr Bull 32: 378-395.

29. Bond GR, Salyers MP, Dincin J, Drake RE, Becker DR, et al. (2007) A randomized controlled trial comparing two vocational models for persons with severe mental illness. J Consult Clin Psychol 75: 968-982. [Crossref]

30. Burns T, Catty J, Becker T, Drake RE, Fioritti A, et al. (2007) The effectiveness of supported employment for people with severe mental illness: A randomised controlled trial. Lancet 370: 1146-1152.

31. Wong KK, Chiu R, Tang B, Mak D, Liu J, et al. (2008) A randomized controlled trial of a supported employment program for persons with long-term mental illness in Hong Kong. Psychiatr Serv 59: 84-90.

32. Kilian R, Lauber C, Kalkan R, Dorn W, Rössler W, et al. (2012) The relationships between employment, clinical status, and psychiatric hospitalisation in patients with schizophrenia receiving either IPS or a conventional vocational rehabilitation programme. Soc Psychiatry Psychiatr Epidemiol 47: 1381-1389.

33. Drake RE, Frey W, Bond GR, Goldman HH, Salkever D, et al. (2013) Assisting social security disability insurance beneficiaries with schizophrenia, bipolar disorder, or major depression in returning to work. Am J Psychiatry 170: 1433-1441. [Crossref]

34. Kukla M, Bond GR (2013) A randomized controlled trial of evidence-based suppor-ted employment: Nonvocational outcomes. J Vocat Rehabil 38: 91-98.

35. Hoffmann H, Jäckel D, Glauser S, Mueser KT, Kupper Z (2014) Long-term effectiveness of supported employment: 5-year follow-up of a randomized controlled trial. Am J Psychiatry 171: 1183-1190. [Crossref]

36. Bejerholm U, Areberg C, Hofgren C, Sandlund M, Rinaldi M (2015) Individual placement and support in Sweden: A randomized controlled trial. Nord J Psychiatry 69: 57-66. [Crossref]

37. Wallace CJ, Tauber R (2004) Supplementing supported employment with work place skills training. Psychiatr Serv 55: 513-515. [Crossref]

38. Mueser KT, Aalto S, Becker DR, Ogden JS, Wolfe RS, et al. (2005) The effective-nes of skills training for improving outcomes in supported employment. Psychiatr Serv 56: 1254-1260. [Crossref]

39. McGurk SR, Twamley EW, Sitzer DI, McHugo GJ, Mueser KT (2007) A metaanalysis of cognitive remediation in schizophrenia. Am J Psychiatry 164: 1791-1802. [Crossref]

40. Tsang HWH, Angell B, Corrigan PW, Lee YT, Shi K, et al. (2007) Cross-cultural study on employers' concerns about hiring people with psychotic disorder: Implica-tions for recovery. Soc Psychiatry Psychiatr Epidemiol 42: 723-733. [Crossref]

41. Bell MD, Zito W, Greig T, Wexler BE (2008) Neurocognitive enhancement therapy with vocational services: Work outcomes at two-year follow-up. Schizophr Res 105: 18-29. [Crossref]

42. Van Rijn H, Borst JP, Taatgen NA, Van Maanen L (2016) On the necessity of integrating multiple levels of abstraction in a single computational framework. Curr Opin Behav Sci 11: 116-120.

43. McLeod CB, Hall PA, Siddiqi A, Hertzman C (2012) How society shapes the health gradient: Work-related health inequalities in a comparative perspective. Annu Rev Public Health 33: 59-73. [Crossref]

44. Fairweather GW, Sanders DH, Maynard H, Cressler DL (1969) Community life for the mentally ill: An alternative to institutional care. Chicago, IL: Aldine.

Copyright: $(2020$ Pulido FR. This is an open-access article distributed under the terms of the Creative Commons Attribution License, which permits unrestricted use, distribution, and reproduction in any medium, provided the original author and source are credited. 